\title{
Retrospective immediate post-operative comparative study of sequential bilateral total knee replacement versus unilateral total knee replacement for knee primary osteoarthritis
}

\author{
Sanil G. Kamat*, Rohan Dessai \\ ${ }^{1}$ Department of Orthopaedics, The Princess Alexandra Hospital NHS trust, United Kingdom \\ ${ }^{2}$ Department of Orthopaedics, Manipal Hospital, Goa, India
}

Received: 21 November 2020

Revised: 05 January 2021

Accepted: 06 January 2021

*Correspondence:

Dr. Sanil G. Kamat,

E-mail: kamat.sanil@gmail.com

Copyright: $\odot$ the author(s), publisher and licensee Medip Academy. This is an open-access article distributed under the terms of the Creative Commons Attribution Non-Commercial License, which permits unrestricted non-commercial use, distribution, and reproduction in any medium, provided the original work is properly cited.

\begin{abstract}
Background: The study is to compare the immediate post operative outcomes of sequential bilateral versus unilateral total knee replacement (TKR) for the treatment of primary knee osteoarthritis. Study comprised of 96 cases of tricompartmental knee primary osteoarthritis who have undergone unilateral and sequential bilateral total knee arthroplasty at Manipal Hospital, Goa from January 2016 to December 2018. The observations for each group was analysed and duration of hospital stay, post operative mobilisation, fall in haemoglobin level immediate post operative, need for blood transfusion, post operative complications, post operative pain and duration of surgery was recorded. The results were statistically compared. The mean duration of hospital stay, post operative blood loss in terms of fall in HB, post operative pain control, need of transfusions and duration of surgery revealed statistically significant differences.

Methods: Total 96 patients diagnosed with primary tricompartmental osteoarthritis were divided into two groups retrospectively. Group 1 operated with sequential bilateral TKR under single anaesthetic procedure and group 2 with unilateral TKR both operated by same surgeon and anaesthetist.

Results: It was observed that longer duration of surgery and hospital stay, higher fall in HB levels, increased need of analgesics and higher requirement of blood transfusions were associated with group 1 as compared to 2. Complication rates and post op mobilisation was similar in both groups.

Conclusions: Sequential bilateral TKR is a viable option for patient with symptomatic bilateral knees but patient selection and pre op counselling takes the priority.
\end{abstract}

Keywords: Total knee replacement, Primary osteoarthritis, Sequential

\section{INTRODUCTION}

Osteoarthritis (OA) is the most common progressive degenerative disease involving the hyaline cartilage covered joints. The knee is the most commonly involved joint and the progression of the disease leads to considerable disability and functional capacity limitation during various spectrum of activities of daily life. Within the aging process, OA becomes a major health problem because of biological dysfunctions. ${ }^{1,2}$ Various treatments have been suggested for symptom re duction and function improvement in these patients. Total knee replacement (TKR) is currently the procedure of choice in advanced stages of OA. Considerable pain reduction and improvement in function and Quality of life (QOL) besides low rate of comorbidity have led to increasing popularity of the procedure. ${ }^{3}$ 
Most patients exhibit degenerative changes in both their knees. A research study revealed that most patients with knee OA need bilateral TKR. ${ }^{4}$ Also, a recent study has suggested that OA progression in the contralateral limb after unilateral total knee arthroplasty (TKA) may be related to altered and asymmetrical movement patterns that overload the contralateral joints. ${ }^{5}$ When the patients need a second operation for their next knee, there are considerations for the repetition of the surgery procedure and anaesthesia side-effects and a second rehabilitation process. ${ }^{5}$ As there is a high rate of indication for bilateral operation, the choice of sequential or unilateral (consecutive) TKR is a challenge for Surgeons and patients. There is a wide range of controversial evidence indicating the preference of unilateral, lack of preference and in favour of sequential bilateral procedure.

This study is mainly performed to compare unilateral versus sequential bilateral TKR in terms of immediate postoperative outcomes.

\section{Aims and objectives}

The objectives of the study were to compare immediate post operative period (from day of surgery to discharge from hospital) of unilateral versus bilateral TKR in terms of: fall in $\mathrm{Hb}$, need for blood transfusion, post operative complication, post operative pain, hospital stay, duration of surgery and immediate post op rehabilitation.

\section{METHODS}

\section{Source of data}

The retro-prospective study was conducted in a tertiary institute by collecting data of 96 cases of tricompartmental knee primary osteoarthritis who have undergone unilateral and sequential bilateral total knee arthroplasty at Manipal Hospital, Goa from January 2016 to December 2018.

The data collected from Medical Record Department of Manipal Hospital. Data was statistically analysed using Statistical package for social sciences (SPSS) 20 version to reach a conclusion. Analysis is descriptive with limitation as patients were analysed from the time of surgery till the time of discharge only.

Ethical approval not required as its an retrospective study and patients personal information not utilised.

All cases operated by single surgeon with same techniques and same anaesthesia protocol including same anaesthetist and same post op protocol including same drug for DVT prophylaxis (Rivaroxaban).

\section{Study design}

This is a comparative retrospective study in which patients were admitted and operated at Manipal Hospital, Goa from January 2016 to December 2018.
The comparison in terms of: duration of hospital stay, postoperative mobilisation for both group of patients, fall in haemoglobin level immediate post operative, need for blood transfusion, post operative complications, post operative pain and duration of surgery.

All patients underwent TKR by cemented, medial parapatellar, and posterior cruciate ligament substituting method performed by a single orthopaedic surgeon. All patients received rehabilitation after the surgery including pain management, exercises for Range of motion (ROM) re-gaining and muscular strength enhancement. The exercises majorly included active ankle pumping for deep venous thrombosis prevention and oedema control, active and active-assisted ROM exercises of knee flexion and extension, strengthening knee joint musculature with special emphasis on knee extensors by isometric method progressing to isotonic exercise and gait education with walker and cane. Exercise performance was supervised by a physiotherapist. Epidural anaesthesia was used in all patients and the infusion was retained post operatively for pain relief. Post operative DVT prophylaxis with Rivaroxaban.

\section{Inclusion criteria}

Surgically fit patients more than 50 years of age who has been diagnosed as having tri-compartmental primary osteoarthritis knee.

\section{Exclusion criteria}

Known RA of knee, complicated surgeries with need for computer navigation, age less than 50 years, patients with pre-operative $\mathrm{Hb}$ levels less than 10 and revision TKRs.

\section{RESULTS}

Overall, majority of patients were females with 80.2 percent whereas male patients constituted only 19.8 percent. This discrepancy was even more evident in Bilateral TKR patients with $96.5 \%$ females versus $3.5 \%$ males. Average age of patient undergoing unilateral TKR was 63.88 years. and in Bilateral TKR was 65.22 years. and the difference was not statistically significant.

Furthermore, duration of surgery in bilateral TKR was higher as compared to unilateral TKR. But per knee duration was considerably lower in bilateral TKR at 120.8 mins as compared to unilateral TKR at 138.90 which is mainly due to less time taken for draping and preparation, repetition of sizes of implant and ease of operating the 2 nd knee after the 1 st and simultaneous closer of one knee and starting of procedure on the other knee (which requires extra assistant on table as compared to unilateral knee).

Average hospital stay for bilateral TKR group was slightly higher than unilateral TKR group although the difference is not statistically significant. In addition to this, Fall in haemoglobin levels was significantly higher in bilateral tkr 
group as compared to unilateral tkr group. Also need for blood transfusion was higher in bilateral groups although the data is not statistically significant. The overall complication rate in both groups was very minimal with no significant difference between the two.

The surgical day epidural infusion rate was slightly higher in bilateral TKR group but was not statistically significant. The day 1 epidural infusion rate was higher in Bilateral group and was statistically highly significant. Epidural infusion was extended onto the 2 nd post operative day in bilateral Groups and was statistically significant. The dosage of systemic Analgesics (injection PCM and injection tramadol) was higher in bilateral group as compared to unilateral group on day0, day 1 and day 2 and was statistically very highly significant.

Use of oral pain killers in Bilateral group was higher than unilateral group and the difference was statistically significant. All patients were given immediate bedside mobilisation although in successive post op days unilateral TKRs were easier to mobilise as compared to bilateral group but the data is not statistically significant.

\section{DISCUSSION}

Our study suggests no significant difference in the age group of patients undergoing unilateral or simultaneous bilateral TKR which is in contrary to past study by Odum et al and Stefansdottir et al which demonstrated that simultaneous bilateral group had patient in lower age group as compared to unilateral TKR. ${ }^{15,17}$

Fall in haemoglobin levels was noted to be significantly higher in the bilateral simultaneous group, as compared to the unilateral group. Prior studies have demonstrated that haematological changes are associated with simultaneous bilateral TKR, which show a relative thrombocytopenia that peaked on second postoperative day. ${ }^{7}$ This change in the clotting mechanism may be the reason for increased loss of blood.

Increased loss of blood in the simultaneous group was responsible for increased requirements of postoperative blood transfusions. This leads to increased risk of transfusion reactions, clotting abnormalities and infection. Brotherton et al found blood loss same for unilateral and simultaneous procedures. ${ }^{8}$ McLaughlin et al found blood loss to be less in the simultaneous group. ${ }^{9}$ However, our experience in this study has consistently demonstrated an increased blood loss in the simultaneous bilateral group, which was probably due to altered coagulation cascade. In addition to this, studies have also suggested more transfusion rate with simultaneous BTKA than with unilateral BTKA which may have resulted from greater blood loss from the bilateral bone cuts and surgical trauma with simultaneous BTKA, which is consistent with the previous observation of lower blood loss after staged BTKA than after simultaneous BTKA - Forester et al, Yoon et al, Bullock at al. ${ }^{14,20,24}$
Higher pain in Bilateral TKR group along with greater dosage of Epidural infusion, systemic and Oral analgesics was demonstrated in our study which is similar to a study in the past by Wang et al. ${ }^{26}$ However, a recent research by Huang et al suggests no significant difference between Unilateral and simultaneous bilateral groups in terms of pain and functional outcomes. ${ }^{25}$

Total surgical time for one knee in the bilateral simultaneous group was 120.8 minutes and it was 138.90 minutes in the unilateral group. This finding is similar to the other studies. Jankiewicz et al reported significant less time with simultaneous group when compared to staged group. ${ }^{10}$ Some advantages of the simultaneous bilateral TKR were single anaesthesia, single preoperative workup and single operating room set up.

Average time of hospital stay was 5.68 days for simultaneous bilateral group and 5.18 days for unilateral group. Hence, one can get both their knees operated in the same time frame as one knee, which could be valuable in a busy hospital centre where beds are always scarce. Findings were similar to a recent study by Eric et al. ${ }^{13}$

Fat embolism has been reported to be consistently low in the literature. ${ }^{11}$ Freeman reported only 2 cases of fat embolism syndrome with 80 procedures. $^{12}$ Our result demonstrated no case of fat embolism syndrome and other systemic complications which was also similar to the findings from the study by $\mathrm{Hu}$ et al, Fu et al, Hussain et al, Ritter et al, Jenny et al, Hooper et al. ${ }^{21,22,23,27,28}$ However a past study by Restrepo et al and Stefansdotir et al have suggested higher cardiac and pulmonary complications associated with simultaneous bilateral TKR. ${ }^{15,16}$

Our study also did not come across any case of superficial or deep knee infection which is similar to the findings from literature by Yoon et al, Fu et al. ${ }^{14,21}$ However another study by Meehan et al shows that infection rate in simultaneous bilateral TKR is lower than unilateral or staged TKR. ${ }^{18}$

Our study has depicted a post op delayed and cumbersome rehabilitation process in cases of simultaneous bilateral TKR which is in coherence to past studies by Lombardi et al and Cushner et al.

Clinical results were identical in both the groups at the time of discharge, but further study is required to determine the long term differences in the two procedures.

\section{CONCLUSION}

The duration of surgery in bilateral TKRs is higher as compared to unilateral TKR but per knee duration is lower. The average hospital stay for both the groups is similar and hence patient can get operated for both knees within the time taken for a single knee with no added complications. Significantly higher blood loss and need for blood transfusion in bilateral groups as compared to unilateral 
TKR and hence it is wiser to select patients for bilateral TKR with $\mathrm{Hb}$ at $12.5 \mathrm{gm} / \mathrm{dl}$ and higher. Post operative pain in bilateral group is significantly higher as compared to unilateral group with higher requirement of analgesic drugs in bilateral TKR cases. Post operative mobilisation is easier and more effective in unilateral TKR patients which may be due to higher pain and extension of epidural infusion in bilateral cases. Hence our study infers that sequential bilateral TKR is a viable option for patient with symptomatic bilateral knees but patient selection and pre op counselling takes the priority.

\section{Funding: No funding sources}

Conflict of interest: None declared

Ethical approval: The study was approved by the institutional ethics committee

\section{REFERENCES}

1. McAlindon TE, Cooper C, Kirwan JR, Dieppe PA. Determinants of disability in osteoarthritis of the knee. Ann Rheum Dis. 1993;52(4):258-62.

2. Boutron I, )Rannoud M,)Jardinaudlopez M.†Meric G)Revel M)Poiraudeau S). Disability and quality of life of patients with knee or hip osteoarthritis in the primary care setting and factors associated with general practitioners' indication for prosthetic replacement within 1 year. Osteoarthritis and Cartilage. 2008;16(9):1024-031.

3. Rönn K, Reischl N, Gautier E, Jacobi M. Current Surgical Treatment of Knee Osteoarthritis. Arthritis. 2011;2011:454873.

4. Metcalfe AJ, Andersson MLE, Goodfellow R, Thorstensson CA .Is knee osteoarthritis a symmetrical disease? Analysis of a 12 year prospective cohort study. BMC Musculoskelet Disord. 2012;13:153.

5. Zeni J, Flowers P, Bade M, Cheuy V. Stiff knee gait may increase risk of second total knee arthroplasty. Journal Of Orthopaedic Research. 2018.

6. Mirghasemi SA, RasouliM MR, Maltenfort S, Rashidinia J, Parvizi. Staged bilateral total knee arthroplasty: when is the second knee safe to be replaced?. Orthopaedic Proceedings. 2018.

7. Stern SH, Sharrock N. Hematologic and circulatory changes associated with total knee arthroplasty surgical instrumentation. Clin Orthop. 1994;299:179.

8. Botherton SL, Roberson JR. Staged versus simultaneous bilateral total knee replacement. J Arthroplasty. 1986;1:221.

9. McLaughlin TP, Fisher RL. Bilateral Total knee arthroplasties. Comparison of simultaneous (two team), sequential and staged knee replacements. Clin Orthop. 1985;199:220.

10. Jankiewicz JJ, Sculco TP. One stage versus 2 stage bilateral total arthroplasty. Clin Orthop. 1994;309:94.

11. Arden GP. Total knee replacement. Clin Orthop. 1993;294:92.
12. Freeman PA. Walldius arthroplasty. A review of 80 cases Clin Orthop. 1973;94:85.

13. Bohm E, Molodianovitsh K, Dragan K, Zhu N, Webster G, Masri B, Schemitsch E, Dunbar M. Outcomes of unilateral and bilateral total knee arthroplasty in 238,373 patients. Acta Orthop. 2016;87(Suppl 1):24-30.

14. Yoon H S, Han C D, Yang I H. Comparison of simultaneous bilateral and staged bilateral total knee arthroplasty in terms of perioperative complications. J Arthroplasty. 2010;25(2):179-85.

15. Stefánsdóttir A, Lidgren L, Robertsson O. Higher early mortality with simultaneous rather than staged bilateral TKAs: results from the Swedish Knee Arthroplasty Register. Clin Orthop Relat Res. 2008;446(12):3066-70.

16. Restrepo C, Parvizi J, Dietrich T, Einhorn $\mathrm{T}$ A. Safety of simultaneous bilateral total knee arthroplasty. A meta-analysis. J Bone Joint Surg (Am). 2007;89(6):1220-6.

17. Odum S M, Troyer J L, Kelly M P, Dedini R D, Bozic K J. A cost-utility analysis comparing the costeffectiveness of simultaneous and staged bilateral total knee arthroplasty. J Bone Joint Surg (Am). 2013;95(16):1441-9.

18. Meehan J P, Danielsen B, Tancredi D J, Kim S, Jamali A A, White R H. A population-based comparison of the incidence of adverse outcomes after simultaneous-bilateral and staged-bilateral total knee arthroplasty. J Bone Joint Surg (Am). 2011;93(23):2203-13.

19. Lombardi A V, Mallory T H, Fada R A, Hartman J F, Capps S G, Kefauver C A, Dodds K, Adams J B.Simultaneous bilateral total knee arthroplasties: who decides? Clin Orthop Relat Res. 2001;(392):319-29.

20. Forster M C, Bauze AJ, Bailie A G, Falworth M S, Oakeshott R D. A retrospective comparative study of bilateral total knee replacement staged at a one-week interval. J Bone Joint Surg (Br). 2006;88(8):1006-10.

21. Fu D, Li G, Chen K, Zeng H, Zhang X, Cai Z. Comparison of clinical outcome between simultaneous-bilateral and staged-bilateral total knee arthroplasty: a systematic review of retrospective studies. J Arthroplasty. 2013;28(7):1141-7.

22. Hu J, Liu Y, Lv Z, Li X, Qin X, Fan W. Mortality and morbidity associated with simultaneous bilateral or staged bilateral total knee arthroplasty: a metaanalysis. Arch Orthop Trauma Surg. 2011;131(9):1291-8.

23. Hussain N, Chien T, Hussain F, Bookwala A, Simunovic N, Shetty V et al. Simultaneous versus staged bilateral total knee arthroplasty: a metaanalysis evaluating mortality, peri-operative complications and infection rates. HSS J. 2013;9(1):50-9.

24. Bullock D P, Sporer S M, Shirreffs T G Jr.Comparison of simultaneous bilateral with unilateral total knee arthroplasty in terms of 
perioperative complications. J Bone Joint Surg (Am). 2003;85(10):1981-6.

25. Yu-Hao Huang, Chin-lin, Jia-Hwa Yang, Chin-Yuan Mou. No difference in the functional improvements between unilateral and bilateral total knee replacements. BMC Musculoskeletal Disorders. 2018.

26. Wang YC, Teng WN, Kuo IT, Chang KY, Chang WK, Tsou MY et al. Patient-machine interactions of intravenous patient-controlled analgesia in bilateral versus unilateral total knee arthroplasty: a retrospective study. J Chin Med Assoc. 2013;76(6):330.

27. Ritter MA, Harty LD, Davis KE, Meding JB, Berend M. Simultaneous bilateral, staged bilateral, and unilateral total knee arthroplasty. A survival analysis. J Bone Joint Surg Am. 2003;85-a(8):1532.
28. Jenny JY, Trojani C, Prudhon JL, Vielpeau C, Saragaglia D, Houillon C et al. Simultaneous bilateral total knee arthroplasty. A multicenter feasibility study. Orthop Traumatol Surg Res. 2013;99(2):191.

29. Hooper GJ, Hooper NM, Rothwell AG, Hobbs T. Bilateral total joint arthroplasty: the early results from the New Zealand National Joint Registry. J Arthroplast. 2009;24(8):1174.

Cite this article as: Kamat SG, Dessai R.

Retrospective immediate post-operative comparative study of sequential bilateral total knee replacement versus unilateral total knee replacement for knee primary osteoarthritis. Int J Res Orthop 2021;7:189-93. 